Europhys. Lett., 26 (6), pp. 425-430 (1994)

\title{
Evidence for Enhanced Electrostatic Interactions in Lamellar Monolayer Systems.
}

\author{
W. K. KegeL $\left(^{*}\right)$ and H. N. W. LeKKERKERKER \\ Van't Hoff Laboratory, University of Utrecht \\ Padualaan 8, 3584 CH Utrecht, The Netherlands \\ (received 6 September 1993; accepted in final form 5 April 1994) \\ PACS. 05.40 - Fluctuation phenomena, random processes, and Brownian motion. \\ PACS. 41.10D - Electrostatics, magnetostatics. \\ PACS. 61.30E - Experimental determinations of smectic, nematic, cholesteric, and lyotropic \\ structures.
}

\begin{abstract}
The interlamellar spacing as a function of the surfactant concentration in a lamellar monolayer system containing a ionic surfactant was studied. It is demonstrated that even at large interlamellar spacings $(6-14 \mathrm{~nm}$ ) relative to the Debye lengths (order $0.7 \mathrm{~nm}$ ), electrostatic repulsion still plays a significant role which is attributed to a coupling with monolayer undulations.
\end{abstract}

Systems consisting of water, oil and one or more amphiphiles may order themselves into a stack of undulating monolayers or bilayers. Over the last decade it has become clear that undulations may strongly renormalize direct interactions arising from intermolecular forces [1-4]. Thermal undulations are predicted to lead to a much slower decay of the electrostatic repulsion between charged bilayer membranes than expected from classical double-layer theory [5-8]. Supporting experimental evidence for such an effect can be obtained from the distance dependence of the interaction between charged membranes [9]. Moreover, Odijk[10] deduced from his self-consistent theory of undulation-enhanced interactions a melting rule for a lamellar phase coexisting with an isotropic phase which is in semi-quantitative agreement with experimental results of Dubois and Zemb [11].

The aim of the work presented here is to provide experimental evidence for enhanced electrostatic repulsion in single-phase fluctuating monolayer systems in which the interlamellar spacing is measured as a function of the monolayer volume fraction. The effective salt concentration in these systems is shown to depend upon the total area of the charged monolayer at the oil-brine interface. We present a simple method to control the effective salt concentration independent of the monolayer volume fraction and show that even at relatively high effective salt concentrations (up to $0.3 \mathrm{M}$ ), electrostatic repulsion still plays a significant role.

We first summarize some theoretical aspects of charged undulating membrane systems that are relevant for the work presented here. In a fluctuating membrane system thermal undulations tend to decrease the projected area while the total area $A$ is conserved. The first-order correction to the projected area $A_{\text {proj }}$ of a membrane caused by thermal

(*) Present address: Department of Chemistry and Biochemistry, University of California, Los Angeles, CA 90024-1569, USA. 
undulations is [12]

$$
\frac{A}{A_{\text {proj }}}=1+\frac{k_{\mathrm{B}} T}{4 \pi K} \ln \left[\frac{q_{\max }}{q_{\min }}\right],
$$

where $K$ denotes the bending elastic modulus. The upper and lower wave number cut-off $q_{\max }$ and $q_{\text {min }}$ are given by

$$
q_{\max }=2 \pi / a, \quad q_{\min }=2 \pi / \lambda,
$$

in which $a$ is a molecular length scale and $\lambda$ the deflection length,

$$
\lambda=c^{\prime} u\left(K / k_{\mathrm{B}} T\right)^{1 / 2},
$$

where $c^{\prime}$ is a constant of order unity $[12,13]$ and $u$ is the root mean square of the amplitude of the undulations. If the interaction between the membranes is dominated by electrostatic repulsion, the membranes are essentially flat. Then the interlamellar (oil plus water) spacing $D$ varies according to the ideal dilution law

$$
D / 2=\frac{\delta}{\phi_{\mathrm{s}}},
$$

where $\delta$ is the thickness of the surfactant layer and $\phi_{\mathrm{s}}$ is the membrane volume fraction. On the other hand, if the electric potential is screened by a large salt concentration, the membranes approximately behave as if they were uncharged. In that case, $u$ is proportional to $D$ and therefore the deflection length is directly proportional to $D$ as well. This leads to a dilution law of the form $[14,15]$

$$
D \phi_{\mathrm{s}} / 2=A-B \ln \left(\phi_{\mathrm{s}}\right)
$$

in which

$$
A=\delta\left(1+\frac{k_{\mathrm{B}} T}{4 \pi K} \ln \left(\frac{c^{\prime \prime} \delta}{a}\left(K / k_{\mathrm{B}} T\right)^{1 / 2}\right)\right),
$$

and

$$
B=\delta k_{\mathrm{B}} T / 4 \pi K,
$$

where $c^{\prime \prime}$ is again a constant of order unity.

At intermediate salt concentration rather complicated relations between $u, D$ and the Debye length $1 / \kappa$ are predicted [7,8]. Furthermore, the Debye length being determined by the effective salt concentration is in turn a function of the amount of charged membranes in the system. Therefore it is imperative to know the effective salt concentration in order to unravel consequences of dilution (i.e. of varying $D$ ) and of varying salt concentration.

To calculate the effective salt concentration $c$ as a function of the initial salt concentration $c_{\text {init }}$ and the amount of (charged) surfactant in the system, we use the relation

$$
c=c_{\text {init }}-\Gamma_{\text {co-ion }}(A / V),
$$

with $A$ the total charged surface, $V$ the volume of brine in the system and $\Gamma_{\text {co-ion }}$ the surface excess concentration of co-ions. Using Gouy-Chapman double-layer theory, one can show that for a $1: 1$ electrolyte [16]

$$
\Gamma_{\text {co-ion }}=-2 c \kappa^{-1}(p-q+1),
$$

where $\kappa$ is the inverse Debye length, and

$$
p=\frac{|\sigma|}{\left(8 \varepsilon_{0} \varepsilon_{\mathrm{r}} R T_{\mathrm{c}}\right)^{1 / 2}}, \quad q=\left(p^{2}+1\right)^{1 / 2},
$$

in which $\sigma$ is the surface charge density, $\varepsilon_{0} \varepsilon_{\mathrm{r}}$ is the dielectric constant of the medium and $R$ is 


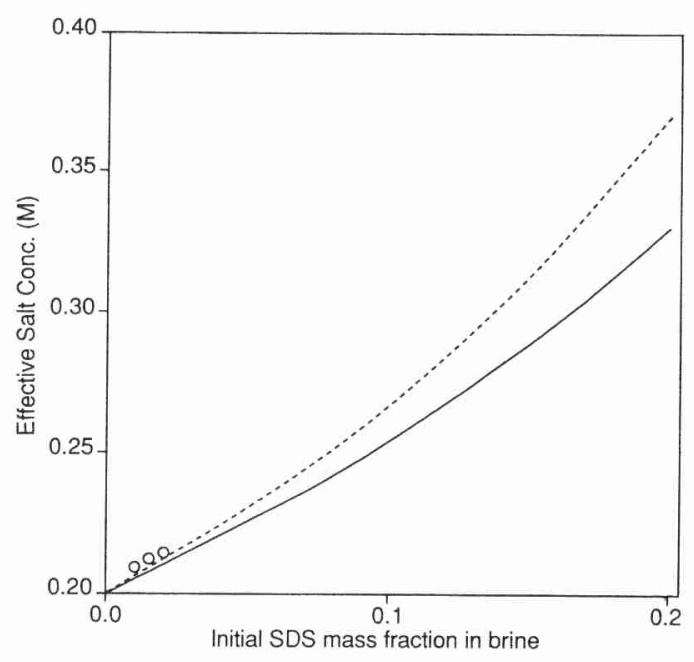

Fig. 1.

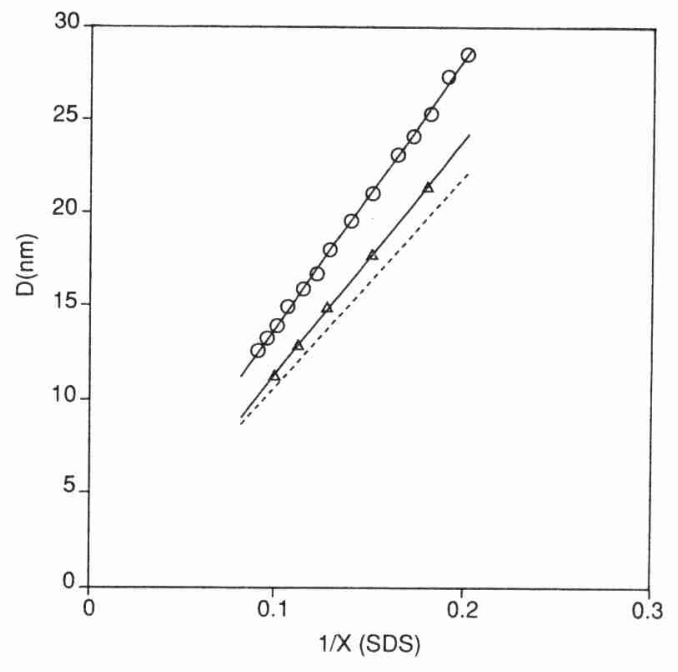

Fig. 2.

Fig. 1. - The effective salt concentration as a function of the initial mass fraction SDS in brine with an initial concentration of $0.20 \mathrm{M}$. Surface charge density calculated according to eq. (12) (solid line) and the upper bound corresponding to an infinite charge density (dashed line). A Stern-layer thickness of $0.2 \mathrm{~nm}$ was used. Points indicate experimental results (see text).

Fig. 2. - Bragg-peak positions $D=2 \pi / q_{\max } v s .1 / x$ (SDS) with $x$ (SDS) the SDS weight fraction (in per cent) in the lamellar phase. Single-phase lamellar systems with constant initial salt concentration (circles) and constant effective salt concentration (triangles) are shown. The dashed line is calculated from the SDS molecular area obtained from interfacial-tension measurements and application of the Gibbs adsorption equation [20].

the gas constant. The effective salt concentration can now be calculated iteratively from (8)-(10) using

$$
A / V=c_{\mathrm{s}} N_{\mathrm{A}} \sigma_{\mathrm{s}}
$$

where $c_{\mathrm{s}}$ denotes the initial (molar) surfactant concentration in brine, $N_{\mathrm{A}}$ Avogadro's number, $\sigma_{\mathrm{s}}$ the surfactant molecular area and

$$
\sigma=-F /\left(N_{\mathrm{A}} \sigma_{\mathrm{s}}\right)
$$

in which $F$ denotes Faraday's constant.

A similar approach was used by Dubois et al. [18] leading to an excellent agreement with the experimentally determined osmotic pressure as a function of the interlamellar spacing in a bilayer system. We checked the calculated effective salt concentration in a system composed of equal volumes of brine $(0.2 \mathrm{M} \mathrm{NaCl}$ initial in water) and cyclohexane, sodium dodecyl sulfate (SDS) and hexanol [19]. The (effective) salt concentration is measured in the excess water phase in a 4-phase equilibrium brine-lamellar phase-microemulsion phase-oil, as a function of the SDS concentration. This system is closely related to the one studied in this letter. The results are presented in fig. 1 (points), together with the calculated effective salt concentrations (lines). The dependence of $\sigma_{\mathrm{s}}$ on the salt concentration is neglegible in the concentration range studied here [17] and a constant SDS molecular area of $0.94 \mathrm{~nm}^{2}$ was used [20]. We moreover introduced an essentially salt-free volume equal to $A \delta_{\mathrm{s}}, \delta_{\mathrm{s}}=0.2 \mathrm{~nm}$ being the thickness of the Stern layer [21,22]. The limiting value $\Gamma_{\text {co-ion }}=-2 c \kappa^{-1}$ corresponding to a very high charge density (eq. (9)) is also indicated in fig. 1 . It seems that the experimentally determined salt concentration is systematically somewhat higher than 
TABLE I. - Composition of the samples with a constant effective salt concentration of $0.20 \mathrm{M}$.

SDS mass fraction Initial salt concentration (M)

Initia pentanol mass fraction in the oil phase

\begin{tabular}{lll}
\hline 0.056 & 0.1530 & 0.2000 \\
0.067 & 0.1422 & 0.2000 \\
0.078 & 0.1311 & 0.2000 \\
0.089 & 0.1195 & 0.2000 \\
0.100 & 0.1076 & 0.2010
\end{tabular}

the calculated concentrations which might be caused by curvature effects in the (coexisting) microemulsion phase [22].

As long as $A \delta_{s} / V \ll 1$, the initial salt concentration necessary to obtain a constant effective salt concentration as a function of the SDS concentration follows from eqs. (8) and (11) with $V$

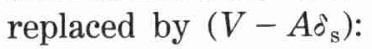

$$
c_{\text {init }}=c+\Gamma_{\text {co-ion }} c_{\mathrm{s}} N_{\mathrm{A}} \sigma_{\mathrm{s}}\left(1+\delta_{\mathrm{s}} c_{\mathrm{s}} N_{\mathrm{A}} \sigma_{\mathrm{s}}\right) .
$$

We studied two kinds of single-phase lamellar systems. In the first system the initial salt concentration was constant and in the other one it was varied in such a way as to keep the effective salt concentration constant as a function of the SDS concentration. The systems studied consisted of equal volumes of brine and oil. The latter was a mixture of cyclohexane and pentanol. The initial mass fraction of pentanol in the oil phase was chosen such that the spontaneous curvature of the surfactant film is about zero (i.e. the pentanol concentration corresponds to a symmetrical position in between two demixing regions in the phase diagram [19]). In the systems with constant initial salt concentration, the partition of pentanol between oil, water and monolayers was kept constant in the way described in [19]. The compositions of the other systems are given in table I.

In fig. 2 the peak positions $D=2 \pi / q_{\max }$ measured by small-angle X-ray scattering (using an Anton Paar Kratky camera) as a function of the inverse surfactant weight fraction in the system is given for the systems with constant initial salt concentration $(0.2 \mathrm{M})$ and constant effective salt concentration $(0.2 \mathrm{M})$. The solid lines are linear best-fit lines. Because of the low intensity of the X-ray source used here, we did not attempt to analyse the peak shapes in order to obtain information concerning the interlayer interactions. Such an analysis was performed in [23] for bilayer systems using synchrotron SAXS. Two interrelated features are apparent from fig. 2 . Firstly, the systems with a constant effective salt concentration all have a lower interlamellar spacing compared to the ones with a constant initial salt concentration at equal SDS concentrations. Secondly, from the slopes of the lines it follows that the molecular areas of SDS are different, being $(0.73 \pm 0.01) \mathrm{nm}^{2}$ for the system with constant initial salt concentration and $(0.84 \pm 0.02) \mathrm{nm}^{2}$ for the system with constant effective salt concentration. The latter value is closer to the value of $0.94 \mathrm{~nm}^{2}$ obtained from interfacial-tension measurements and applying the Gibbs adsorption equation $[20]\left({ }^{1}\right)$. These features are first indications that electrostatic repulsion still plays a role in the system studied here as area is recovered (i.e. the monolayers are «flattened») upon decreasing the salt concentration.

By plotting $D \phi_{\mathrm{s}} / 2 v s .-\ln \left(\phi_{\mathrm{s}}\right)$ (fig. 3), it is apparent that the system with constant initial salt concentration very nearly follows the «ideal dilution» law eq. (4), while the system with

( ${ }^{1}$ It has been recently shown that adsorption densities obtained by interfacial-tension measurements are in agreement with the ones obtained by neutron reflection [24]. 


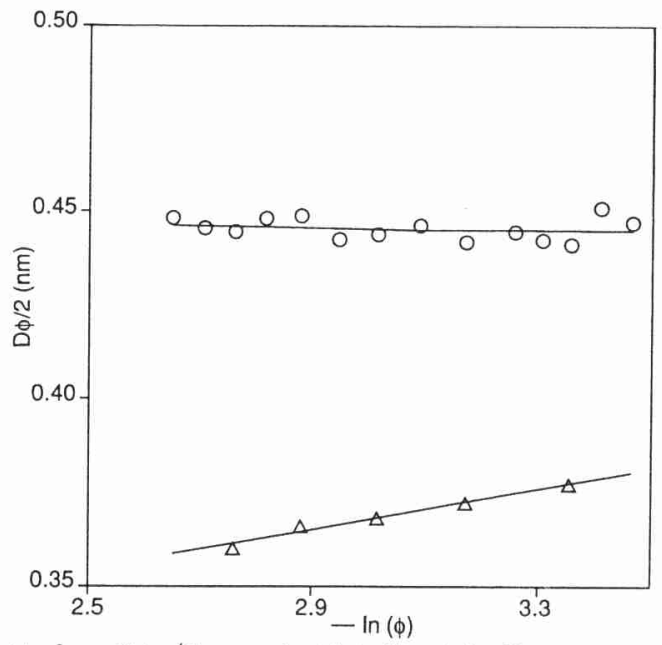

Fig. 3. - Data from fig. 2 plotted as $D \phi_{\mathrm{s}} / 2 v s$. $-\ln \left(\dot{\psi}_{\mathrm{s}}\right)$. Straight lines correspond to a best fit of the data to eq. (5).

constant effective salt concentration has a noticeable deviation from this ideal swelling and can be described by eq. (5) with $B=0.026 \mathrm{~nm}$. This value of $B$ is in good agreement with the one calculated from eq. (7) using $K / k_{\mathrm{B}} T=1$ [25] and $\delta=\bar{v}_{\mathrm{s}} / \sigma_{\mathrm{s}}=0.35 \mathrm{~nm}$, with $\bar{v}_{\mathrm{s}}$ the molecular volume of SDS being $0.33 \mathrm{~nm}^{3}$ [17]. Apparently, the interlamellar spacing in the system with a constant initial salt concentration has no measurable $\ln \phi_{\mathrm{s}}$-dependence even though the amount of lost area is relatively large. On the contrary, the interlamellar spacing in the system with constant effective salt concentration has a significant $\ln \phi_{\mathrm{s}}$-dependence even though its lost area is smaller. This points to the important role of electrostatic repulsion and the confounding influence of salt partitioning. Upon diluting, the interlamellar spacing $D$ is increased, which tends to increase the root-mean-square amplitude of the undulations $u$. This is clear from the results on the system with constant effective salt concentration. In the other system, this trend is apparently compensated by the concomitant change in the effective salt concentration having the opposite effect on $u$. This results in an apparent ideal dilution behaviour caused by two compensating effects. The undulation amplitude is however larger than in the system with constant effective salt concentration, leading to a difference in the projected area of about $15 \%$.

It is tempting to compare our results quantitatively with the available theories of charged undulating membranes where two asymptotic limits are considered. One is attained at relatively low salt concentrations where the membranes are supposed to be highly ordered (the spatial distribution of each membrane is Gaussian) $[8,10]$. In this regime it is expected that small variations of $\kappa$ give rise to relatively large variations of $u$ and, thus, of the interlamellar spacing $D$. The other limit is reached at relatively large membrane separations and/or high salt concentrations. This limit is very close to the Helfrich regime [12], the only difference being the presence of a small boundary layer with an effective thickness of order $1 / \kappa$ which prevents the monolayers from touching each other [10]. From the work of Odijk $[8,10]$ we deduce that the system studied here is (somewhat) beyond the first mentioned regime. The value of $B$ obtained from the slope in fig. 3 of the system with constant effective salt concentration is in good agreement with the calculated value of $B$ (eq. (7)). This agreement seems to imply that (approximately) the Helfrich limit is reached. As $D \gg 1 / \kappa$ in the latter regime, the small variations of $\kappa$ as encountered here (i.e. between 1.4 and $1.9 \mathrm{~nm}^{-1}$ ) will hardly influence $u$. This, however, is in contradiction with the results depicted in fig. 2 and 3 . It is clear from fig. 2 (and fig. 1) that only very small changes in 
effective salt concentration (between approximately $0.05 \mathrm{M}$ and $0.13 \mathrm{M}$ corresponding to differences in $1 / \kappa$ of order $0.1 \mathrm{~nm}$ ) already lead to significant differences in $D$. These differences are attributed to differences in $u$. Moreover, as mentioned above, from fig. 3 it follows that a small decrease of the effective salt concentration compensates the growth in the undulation amplitude due to dilution. A way out of this apparent contradiction is that the systems studied here correspond to the cross-over regime between the two limits mentioned above. At the moment, there is no theory explicitly describing this regime.

To conclude, the experimental results presented provide strong evidence that electrostatic repulsion plays a significant role in single phasic lamellar monolayer systems even at salt concentrations up to $0.3 \mathrm{M}$ and interlamellar spacings of order $10 \mathrm{~nm}$. A decreasing salt concentration tends to «flatten» the monolayers leading to a decreasing (mean) interlamellar spacing.

By compensating for the negative adsorption of salt, it is shown that an apparent ideal behaviour of a lamellar system upon dilution is caused by two compensating effects on the undulation amplitude of the monolayers.

One of us (HL) thanks T. ODIJK for extensive discussions and correspondence on undulationenhanced electrostatic repulsion.

\section{REFERENCES}

[1] Lipowski R. and Leibler S., Phys. Rev. Lett., 56 (1986) 2541.

[2] Leibler S. and Lipowski R., Phys. Rev. Lett., 58 (1987) 1796.

[3] Larche F. C., Appell J., Porte G., Bassereau P. and Marignan J., Phys. Rev. Lett., 56 (1986) 1700.

[4] Evans E., Langmuir, 7 (1991) 1900.

[5] Evans E. A. and Parsegian V. A., Proc. Natl. Acad. Sci. USA, 83 (1986) 7132.

[6] Pincus F., Joanny J. F. and Andelman D., Europhys. Lett., 11 (1990) 763.

[7] Evans E. and IPSEn J., Electroch. Acta, 36 (1991) 1735.

[8] ODIJK T., Langmuir, 8 (1992) 1690.

[9] Loosely-Millman M., Rand R. P. and Parsegian V. A., Biophys. J., 40 (1982) 221.

[10] OdIJK T., submitted to Europhys. Lett.

[11] Dubois M. and Zemb T., Langmuir, 7 (1991) 1352.

[12] Helfrich W. and Servuss R. M., Nuovo Cimento D, 3 (1984) 137.

[13] Golubovic L. and Lubenski T. C., Phys. Rev. B, 39 (1989) 12110.

[14] Strey R., Schomäcker R., Roux D., Nallet F. and Olsson U., J. Chem. Soc. Faraday Trans., 86 (1990) 2253.

[15] Roux D., Nallet F., Freyssingeas E., Porte G., Bassereau P., Skouri M. and Marignan J., Europhys. Lett., 17 (1992) 575.

[16] Overbeek J. Th. G., Prog. Biophys. Biophysical Chem., 6 (1956) 57.

[17] VerhoeckX G. J., De Bruyn P. L. and Overbeek J. Th. G., J. Colloid Interface Sci., 119 (1986) 409.

[18] Dubois M., Zemb T., Belloni L., Delville A., Levitz P. and Setton R., J. Chem. Phys., 96 (1992) 2278.

[19] Kegel W. K. and Lekkerkerker H. N. W., J. Phys. Chem., 97 (1993) 11124.

[20] Kegel W. K., van Aken G. A., Bouts M. N., Lekkerkerker H. N. W., Overbeek J. Th. G. and De BRuYN P. L., Langmuir, 9 (1993) 252.

[21] Biais J., Barthe M., Bourrel M., Clin B. and Lalanne P., J. Colloid Interface Sci., 109 (1986) 576.

[22] van Aken G. A., Overbeek J. Th. G., De Bruyn P. L. and Lekkerkerker H. N. W., J. Colloid Interface Sci., 157 (1993) 235.

[23] Roux D. and Safinya C. R., J. Phys. (Paris), 49 (1988) 307.

[24] Lu J. R., Simister E. A., Thomas R. K. and Penfold J., J. Phys. Chem., 97 (1993) 6024.

[25] Kegel W. K., Thesis, Universiteit Utrecht, Chapter 3 (1993). 